\title{
Significance of ovarian transposition in the preservation of ovarian function for young cervical cancer patients undergoing postoperative volumetric modulated radiotherapy
}

\author{
Hanzi Xu ${ }^{1 \#}$, Chang Guo ${ }^{1 \#}$, Xiuming Zhang ${ }^{2 \#}$, Yaqin $W^{1}{ }^{1}$, Biqing Zhu ${ }^{1}$, Emei Lu ${ }^{1}$, Zhihua Sun ${ }^{1}$, Dan $\mathrm{He}^{1}$, \\ Fei Deng ${ }^{3}$, Juan $\mathrm{Lv}^{4}$, Zhen Gong ${ }^{4}$ \\ ${ }^{1}$ Department of Radiation Oncology, Jiangsu Cancer Hospital, Jiangsu Institute of Cancer Research, The Affiliated Cancer Hospital of Nanjing \\ Medical University, Nanjing, China; ${ }^{2}$ Department of Radiology, Jiangsu Cancer Hospital, Jiangsu Institute of Cancer Research, The Affiliated \\ Cancer Hospital of Nanjing Medical University, Nanjing, China; ${ }^{3}$ Department of Gynecological Oncology, Jiangsu Cancer Hospital, Jiangsu \\ Institute of Cancer Research, The Affiliated Cancer Hospital of Nanjing Medical University, Nanjing, China; ${ }^{4}$ Department of Gynecology, Women's \\ Hospital of Nanjing Medical University, Nanjing Maternity and Child Health Care Hospital, Nanjing, China \\ Contributions: (I) Conception and design: H Xu, C Guo, X Zhang, Z Gong; (II) Administrative support: C Guo, X Zhang; (III) Provision of study \\ materials or patients: H Xu, Y Wu, B Zhu, E Lu, Z Sun, D He, F Deng, J Lv; (IV) Collection and assembly of data: H Xu, C Guo, X Zhang, Z \\ Gong; (V) Data analysis and interpretation: H Xu, Z Gong; (VI) Manuscript writing: All authors; (VII) Final approval of manuscript: All authors. \\ \#These authors contributed equally to this work. \\ Correspondence to: Zhen Gong. No. 123, Tianfei Road, Nanjing 210004, China. Email: gongzhen@njmu.edu.cn.
}

Background: This paper aimed to evaluate the effectiveness of ovarian transposition (OT) and the dose constraint for preserving ovarian function in young cervical cancer patients who underwent postoperative volumetric modulated arc therapy (VMAT).

Methods: A retrospective analysis was conducted of young cervical cancer patients who accepted postoperative VMAT in the Affiliated Cancer Hospital of Nanjing Medical University from September 2015 to September 2018. VMAT plans for OT and non-OT patients were compared, and the patients' ovarian function was followed up. The transposed position of the ovaries and the radiation dose constraint were further explored using a receiver operator characteristic (ROC) curve.

Results: A total of 51 young patients (age $\leq 40$ years) were included in the study, 32 of whom underwent OT and 19 of whom did not. For these OT and non-OT patients, the homogeneity index (HI), conformity index (CI), organs at risk (OARs), average number of monitor units (MUs), and mean treatment time were similar and showed no statistically significant difference $(\mathrm{P} \geq 0.05)$. Through follow-up studies, the number of patients with preserved ovarian function was found to be 22 (out of 32) and 0 (out of 19) in the OT and nonOT patients, respectively. The minimal distance for preserving ovarian function was determined as $2.1 \mathrm{~cm}$ between the center of a transposed ovary and the planning target volume (PTV) margin. The optimal limited radiation doses were estimated as maximum dose $\left(D_{\max }\right) 9.8 \mathrm{~Gy}$ and mean dose $\left(\mathrm{D}_{\text {mean }}\right) 4.6 \mathrm{~Gy}$, respectively.

Conclusions: OT shows no negative effects on dose distribution, target region conformity, protection of OARs, or treatment efficacy and is therefore a reliable method in the preservation of ovarian function for young cervical cancer patients undergoing postoperative radiotherapy using the VMAT technique. Specifically, when the distance between the center of a transposed ovary and the PTV margin is more than $2.1 \mathrm{~cm}$, and the radiation dose is limited to a $\mathrm{D}_{\max }$ of less than $9.8 \mathrm{~Gy}$ and a $\mathrm{D}_{\text {mean }}$ of less than 4.6 Gy, the function of transposed ovaries may be preserved.

Keywords: Cervical cancer; ovarian function; ovarian transposition volumetric modulated arc therapy (ovarian transposition VMAT)

Submitted Jun 05, 2021. Accepted for publication Sep 30, 2021.

doi: 10.21037/atm-21-2909

View this article at: https://dx.doi.org/10.21037/atm-21-2909 


\section{Introduction}

Cervical malignancy is the fourth most common cancer found in women, with approximately 600,000 women worldwide suffering from this disease every year (1). When diagnosed, most of these patients have early-stage disease (I-II) according to the International Federation of Gynecology and Obstetrics (FIGO) criteria, and a considerable proportion are pre-menopausal (2). In the United States, roughly $28 \%$ of estimated new patients are younger than 40 years of age (3). In China, the incidence of younger patients is also increasing, especially in underdeveloped regions (4). For patients with earlystage cervical cancer, the therapeutic effect of surgical procedures is equivalent to that of radiation therapy (5). However, ovaries are highly susceptible to radiation, and external radiation therapy can irreversibly damage ovarian function and lead to ovarian failure, resulting in low-estrogen sequelae, which may include menopausal symptoms, osteoporosis, or even long-term cardiovascular complications (6). Although hormone replacement therapy (HRT) can contribute to the prevention or remission of these risks, HRT itself may lead to serious adverse side effects $(7,8)$. Based on these considerations, young patients with stage $\leq$ IIA cervical cancer in China are strongly recommended to accept radical surgery rather than radiation therapy. However, these postoperative patients, especially those with positive resection margins, uterine invasion, or pelvic or para-aortic lymph node metastasis, are recommended to receive adjuvant irradiation, typically external radiation therapy; thereby, they are still subject to the risk of radiation damage to the ovaries in situ.

With the extremely low incidence of ovarian metastasis for early diagnosed patients, cervical cancer is generally considered to be a hormone-independent tumor. Therefore, ovary retention is a logically safe measure for cervical cancer, including squamous cell carcinoma or even adenocarcinoma and adenosquamous cancer (9).

As the age of patients is lowering, greater concern should be given to the protection of a patient's ovarian function after a complete eradication of malignant tumors. In recent years, surgical methods of transferring the ovary outside the pelvic radiation zone to avoid radiation damage have received increased attention. Three commonly used clinical methods for ovarian preservation exist: ovarian transposition (OT), ovarian burial, and ovarian cryo-conservation. OT was first proposed in 1956 with the purpose of preserving fertility for young women requiring pelvic irradiation $(10,11)$.
This method, which is performed either by laparotomy or laparoscopy, has been widely used with the transposed ovary standard of " $4 \mathrm{~cm}$ outside the radiation target, or at least $1.5 \mathrm{~cm}$ above the iliac crest" $(10,12,13)$. However, these recommendations were designed with reference to the results of $2 \mathrm{D}$ box-technique irradiation $(10,12,13)$ or intensitymodulated radiotherapy (IMRT) $(14,15)$. Recent studies have reported that volumetric modulated arc therapy (VMAT), a special kind of IMRT, is superior to the usual irradiation due to its potential to reduce the dose of radiation delivered to the ovaries (16). In a previous study, we demonstrated the efficiency of VMAT planning in cervical malignancy (17). The present study was designed to investigate the benefits of OT during VMAT for postoperative cervical cancer patients. We present the following article in accordance with the Standards for Reporting Diagnostic Accuracy (STARD) reporting checklist (available at https://dx.doi.org/10.21037/ atm-21-2909).

\section{Methods}

\section{Patients}

This retrospective analysis included 51 patients with cervical cancer who had received postoperative radiation therapy and concurrent chemotherapy in the Department of Radiation Oncology of the Affiliated Cancer Hospital of Nanjing Medical University between September 2015 and September 2018. The clinical data were filtered for further review. The study was conducted in accordance with the Declaration of Helsinki (as revised in 2013) and approved by the ethics committee of the Affiliated Cancer Hospital of Nanjing Medical University (No. 2021-006). Individual consent for this retrospective analysis was waived. The specific indications, as mentioned by Du et al. (18), were as follows: (I) the patients were diagnosed with cancer of the cervix uteri with pathological evidence; (II) the patients were assessed as stage IA2-IIA2 according to the FIGO 2009 criteria, with or without treatment of unilateral or bilateral OT surgery; (III) the patients were aged 40 years and under; (IV) the patients displayed no climacteric symptoms before radiation; and (V) the patients were not receiving HRT.

\section{Radiation therapy}

Consistent with the National Comprehensive Cancer Network (NCCN) guidelines, the radiation therapy (whole 
pelvic external beam radiotherapy) was conducted by experienced radiation oncologists and radiologists after radical hysterectomy with pelvic lymph node dissection. Computed tomography (CT) simulation was conducted as in our previous study, with the difference that the CT images were transferred to the Monaco 5.1 treatment planning system (Elekta AB, Stockholm, Sweden) (17). Following the recommendations of the International Commission on Radiation Units \& Measurements (ICRU) Report 83, the target volumes and organs at risk (OARs) were delineated as in our previous study (17). The prescription dose was DT4500-4512 cGy/24-25 f/5 w, and the OAR dose was as follows: rectum V50 $<20 \%$, V $40<40 \%$; bladder V $50<20 \%$, $\mathrm{V} 40<40 \%$; small bowel maximum dose $\left(\mathrm{D}_{\max }\right)<52 \mathrm{~Gy}$, V $40<50 \%$; femoral head V $50<5 \%$, V $30<30 \%$; and spinal cord $\mathrm{D}_{\max }<40 \mathrm{~Gy}$. All plans were created and optimized according to the Radiation Therapy Oncology Group (RTOG) 0724 protocol by 2 experienced physicists using a 160-multi-leaf collimator (MLC; Elekta AB, Stockholm, Sweden).

The VMAT plan was performed on all patients using two $360^{\circ}$ coplanar arcs. The radiation dose to the transposed ovaries was limited to as low as possible, with unilateral OT patients receiving unilateral limitations and bilateral OT patients receiving bilateral limitations. However, these limitations did not have priority, meaning that the dose constraints for the transposed ovaries would only be performed once the coverage of the planning target volume (PTV) and the OAR requirements were both satisfied. The ovarian recognition and contouring for OT patients were based on the clips left either on top or at the bottom of the ovaries for labeling by the surgeon. In the absence of metal markers, a radiologist was needed to track and identify the transposed ovaries in CT or magnetic resonance imaging (MRI) images. The quality of the above plans was evaluated using the conformity index (CI) and the homogeneity index (HI); the monitor units (MUs), together with the treatment time, were further compared as previously described (17).

\section{Adjuvant therapy}

No brachytherapy was given, but concurrent chemotherapy ( $25 \mathrm{mg} / \mathrm{m}^{2}$ of cisplatin weekly for $4-6$ weeks) was given to all patients.

\section{Follow-up and evaluation}

All patients were followed up before radiation; immediately after radiation; and 3 months, 6 months, and almost 1-year post-irradiation. Patients' ovarian status was determined by assessing the serum sex hormones. The criteria of preserved ovarian function were defined as the serum concentration of follicle-stimulating hormone (FSH) $<40 \mathrm{mIU} / \mathrm{mL}$, estrogen (E2) $>10 \mathrm{pg} / \mathrm{mL}$ without HRT, and no menopausal symptoms (19).

\section{Statistical analysis}

The Student's $t$-test was used to compare the continuous variables, including patient age, body mass index (BMI), and hormone level, which are expressed as mean and standard deviation (SD). Comparisons of clinical stage, histology, and the distribution of patients, etc. were performed using Pearson's chi-square test. The optimum cutoff point of the transposed position and the ovarian dose constraint were calculated using a receiver operating characteristic (ROC) curve. Statistical analysis was completed using the SPSS 19.0 software (IBM Corp., Armonk, NY, USA), and a P value of $<0.05$ was considered statistically significant.

\section{Results}

\section{Population and characteristics}

From September 2015 to September 2018, there were a total of 51 younger cervical cancer patients who were treated concurrently with postoperative VMAT and adjuvant chemotherapy. All patients underwent the standard procedure of radical hysterectomy and/or pelvic lymphadenectomy before VMAT, 32 of whom underwent OT (19 cases by laparoscopy and the remaining cases by laparotomy), but 19 did not. Bilateral OT was conducted on 23 patients (71.9\%), and unilateral OT was conducted on 9 patients (28.1\%). For OT and non-OT patients, the average age was 34.1 and 35.0 years, respectively. According to the 2009 FIGO criteria, there were 22 OT patients at a stage lower than IB2, and 10 patients with stage IIA disease. Among the non-OT patients, 1 was at stage IA2, 14 were at stage IB, and 4 were at stage IIA. Two considerations were noteworthy: (I) a number of patients (either in the OT-group or in the nonOT group) were at the $\leq$ IB1 stage, meaning the potential necessity of postoperative radiotherapy was relatively lower; and (II) there was a likelihood that ovarian insufficiency after OT might appear for some patients even without their receiving postoperative radiotherapy. Therefore, a few patients undertook non-OT or only unilateral OT treatment. 
Table 1 Characteristics of the patients $(\mathrm{N}=51)$

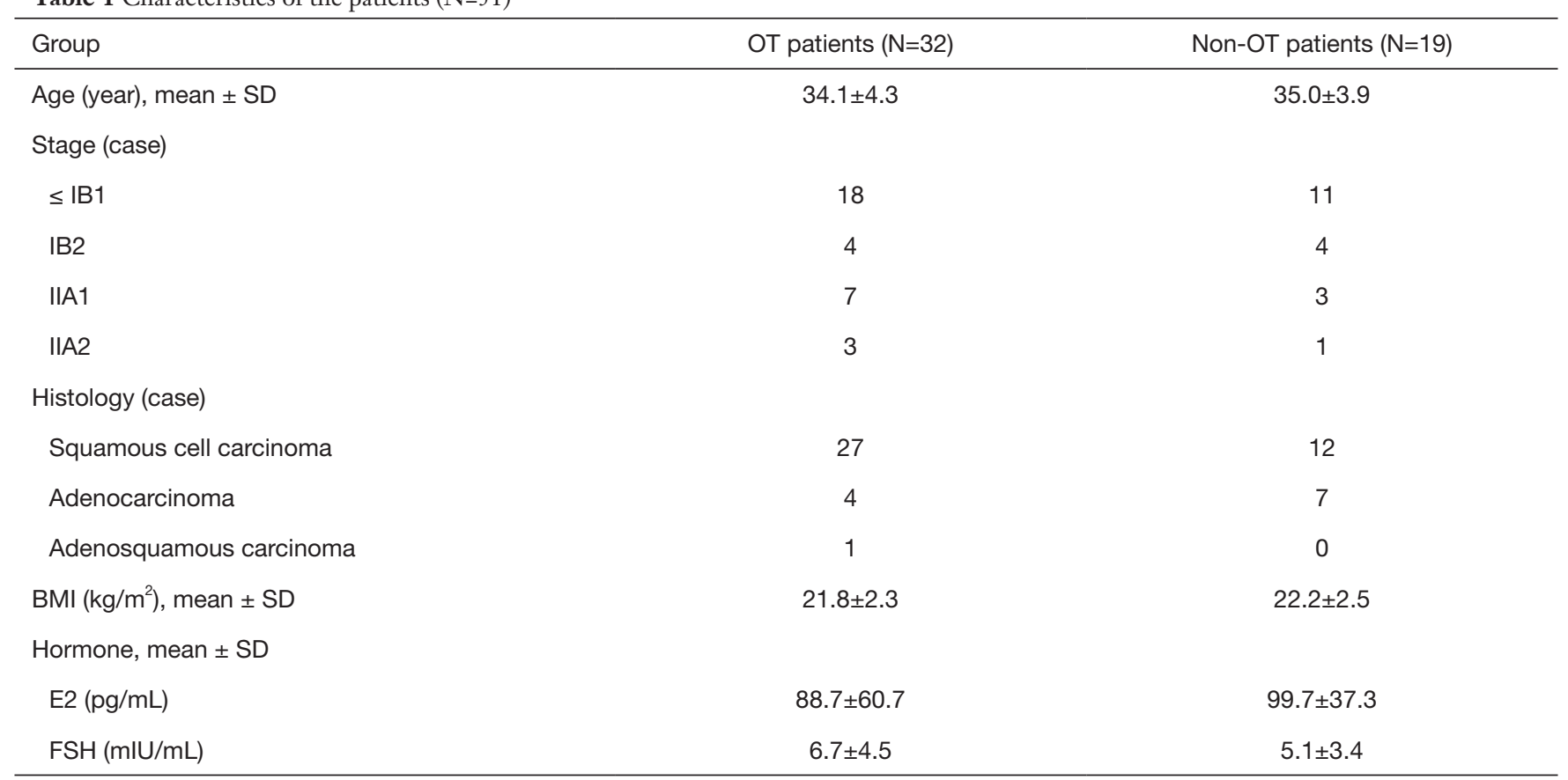

OT, ovarian transposition; SD, standard deviation; BMI, body mass index; E2, estrogen; FSH, follicle-stimulating hormone.

Histologically, 27 cases of squamous cell carcinoma, 4 cases of adenocarcinoma, and 1 case of adeno-squamous carcinoma were identified among OT patients. In the nonOT patients, 12 cases were squamous cell carcinoma and 7 were adenocarcinoma. The characteristics of the 51 patients were summarized, and no significant differences (e.g., in age, stage, histology) between OT and non-OT patients were discovered, as shown in Table $1(\mathrm{P} \geq 0.05)$.

\section{Radiotherapy administration}

The CT planning scans for ovary contouring were included for all patients, although diagnostic MRI scans were only available for a few OT patients. The ovarian position on the CT image was analyzed in advance with treatment planning system. A representative picture is shown in Figure 1. Following the recommendations of ICRU Report 83 , all treatments were successfully administered and well tolerated, and no delay in radiation therapy was observed. The standard plan in OT and non-OT patients is shown in Figure 2.

\section{Comparison of dosimetric parameters}

The dose received by the target volume in the above plans, i.e., $\mathrm{D}_{2 \%}$ (the approximate maximum dose), $\mathrm{D}_{50 \%}$ (the approximate median dose), and $\mathrm{D}_{98 \%}$ (the approximate minimum dose), were compared, and no significant differences were found between the OT and non-OT patients ( $\mathrm{P} \geq 0.05$, see Table 2). The CI and HI, as compared in Table 2, were similar, with no statistical differences observed $(\mathrm{P} \geq 0.05)$. This indicated that the irradiation distribution and the target region conformity had not been affected by the OT patients' plan.

\section{Dose comparison of OARs}

In terms of OARs, such as the bladder, small intestine, and femoral heads, the $V_{10}, V_{20}, V_{30}$, and $V_{40}$ all appeared to be the same, with no statistically significant differences between the OT and non-OT patient plans $(\mathrm{P} \geq 0.05$; see Table 3). This indicated that protection of OARs had not been decreased by the OT patients' plan.

\section{Comparison of treatment efficiency}

The MUs and the total median length for treatment time were also compared to evaluate the performance of the 2 plans. Results showed no statistical differences relating to the average number of MUs $(1,040.5 \pm 102.5 v s .894 .6 \pm 112.1 \mathrm{~s})$ 

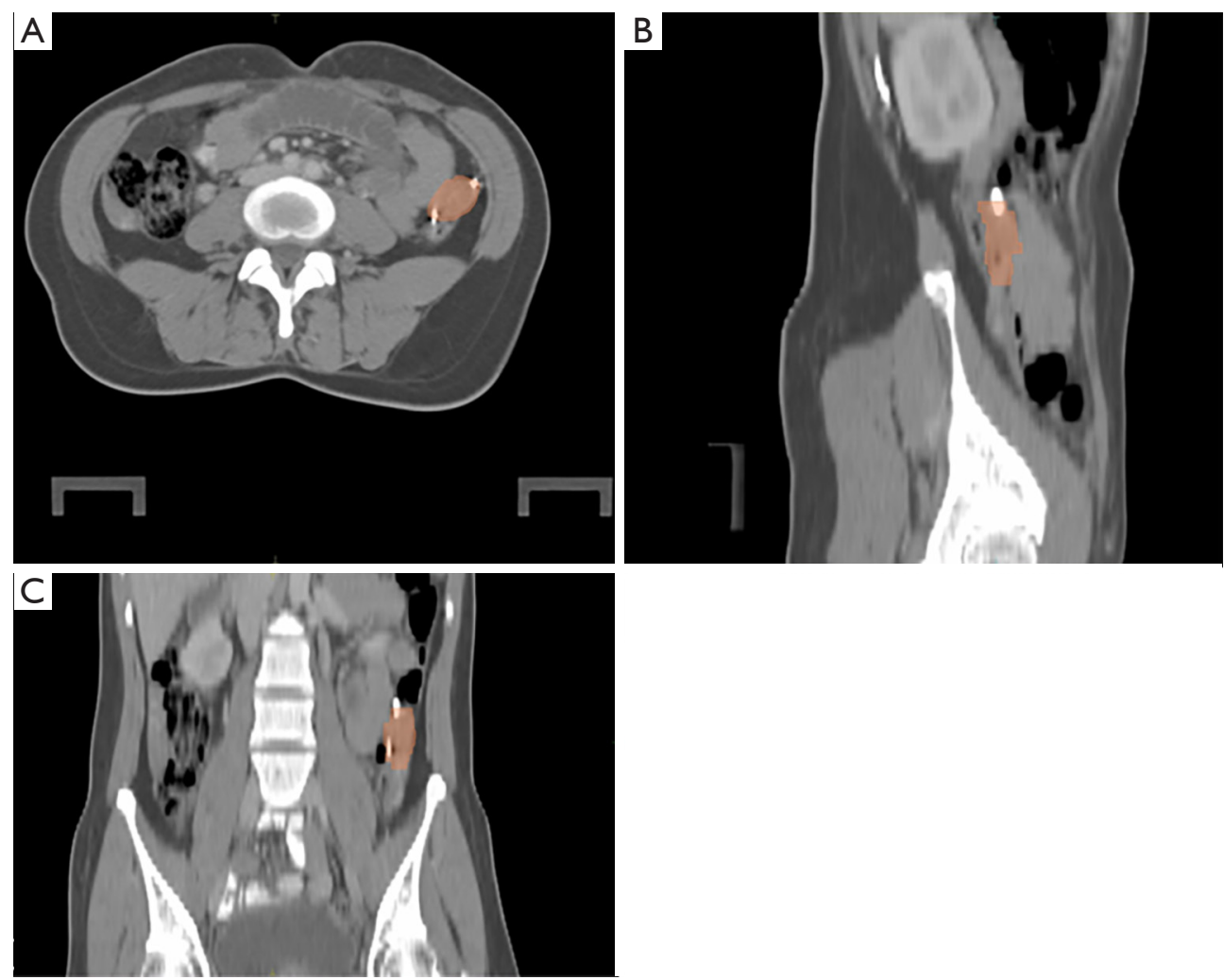

Figure 1 Representative images of ovary identification, showing the left transposed ovary colored orange in axial (A), sagittal (B), and coronal (C) slices.

or the mean treatment time $(33.3 \pm 2.4$ vs. $34.2 \pm 2.7$ days $)$ between the OT and non-OT patients. This indicated that the treatment efficacy had not been reduced for the OT patients (see Table 4).

\section{Comparison of ovarian survival}

After receiving the limited radiation dose in the transposed ovaries, a total of 22 (out of $32 ; 68.8 \%$ ) OT patients tolerated the radiation therapy with preserved ovarian function almost 1 year after the treatment. In contrast, no $(0 / 19)$ ovarian function was found in the non-OT patients during the same time period. The differences regarding ovarian function between the $\mathrm{OT}$ and non-OT patients were significant although no clinical recurrence was detected in either patient group. The optimal length from the center of a transposed ovary to the PTV margin was explored using a ROC curve and Youden's index (the result of each ovary was calculated in bilateral OT patients without ovarian failure), and further calculations were completed based on the ROC curve. The results showed that the minimal distance was at least $2.1 \mathrm{~cm}$ for the prevention of ovarian failure in OT patients. The corresponding area under the curve (AUC) was 0.787 (95\% confidence interval: 0.690-0.865; see Figure 3). Similarly, we also estimated the dose limitation for ovarian function preservation in these patients, and the results showed that the optimal limits were $\mathrm{D}_{\max } 9.8 \mathrm{~Gy}$ and mean dose $\left(\mathrm{D}_{\text {mean }}\right)$ 4.6 Gy. The corresponding AUCs were 0.812 and 0.790, respectively, and the $95 \%$ confidence intervals were $0.717-$ 0.885 and $0.693-0.868$, respectively (Figure 4).

\section{Discussion}

Owing to the continuous advancement of therapy procedures, the rates of cure or complete remission are increasing in young patients with cervical cancer. Therefore, maintaining ovarian function is of increased importance for medical research and for the quality of life of premenopausal patients $(10,20)$. Ovarian conservation was first performed by McCall et al. in 1958 (21) and became widely accepted in radical hysterectomy before irradiation 

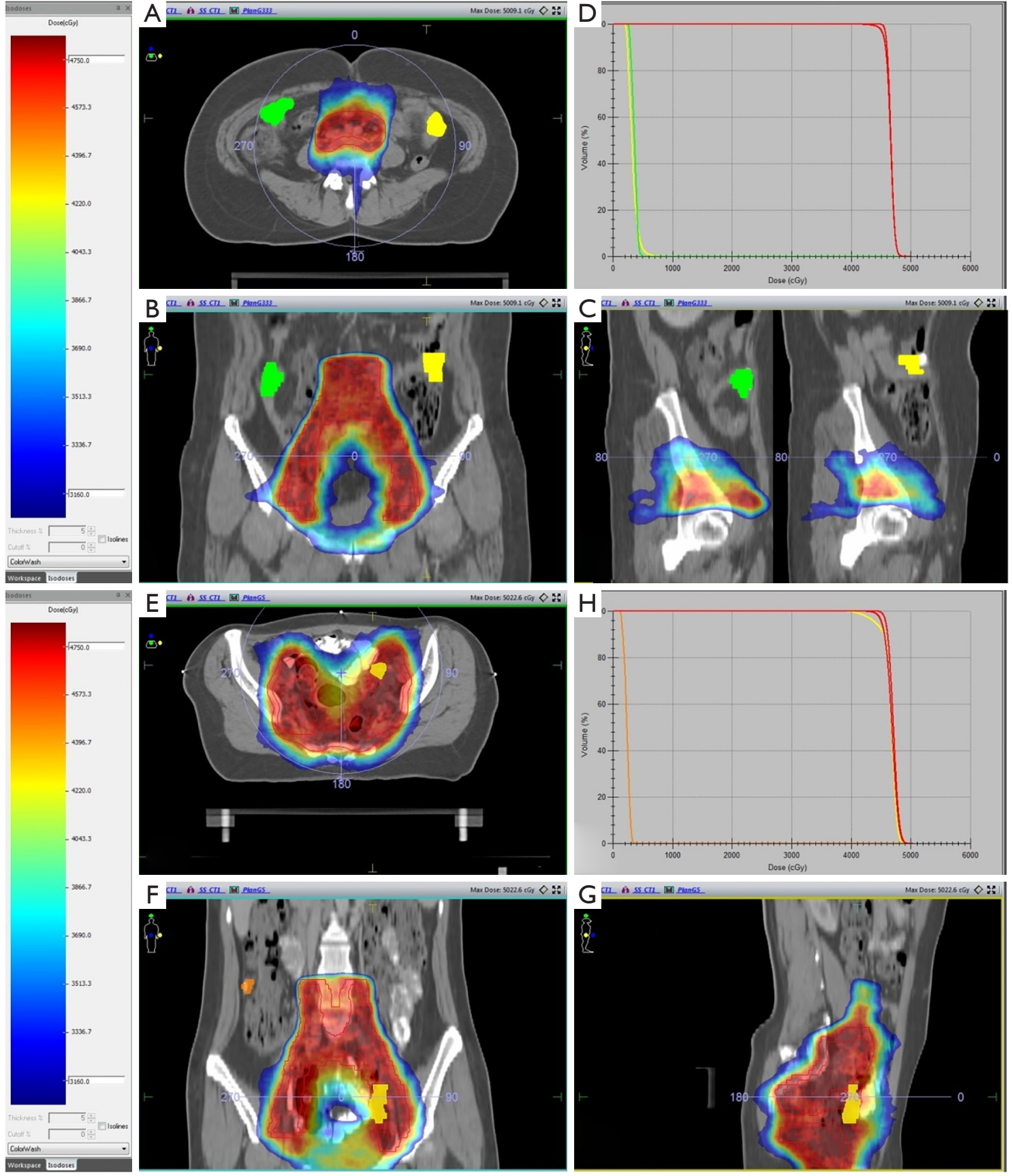

Figure 2 Representative images of the target volume for OT postoperative cervical cancer patients. (A-C) The bilateral transposed ovaries colored green (right) or yellow (left) in axial (A), coronal (B), and sagittal (C) slices. (D) The DVH. (E-G) The unilateral (right) transposed ovarian is colored orange color in axial (E), coronal (F), and sagittal $(\mathrm{G})$ slices. $(\mathrm{H})$ The corresponding DVH. OT, ovarian transposition; DVH, dose-volume histogram.

for cervical cancer (22). However, various factors affect the function of the retained ovaries. In particular, postoperative radiotherapy significantly affects ovarian function, as shown in all previous related studies. This can be prevented by using OT prior to pelvic radiation, a surgical technique which has been used since 1952 with varied degrees of 
Table 2 Comparison of dosimetric parameters between OT and non-OT patients

\begin{tabular}{lccc}
\hline Item & OT patients & Non-OT patients & P value \\
\hline$D_{2 \%}(G y)$ & $48.1 \pm 0.6$ & $47.7 \pm 0.8$ & 0.097 \\
$D_{50 \%}(G y)$ & $46.5 \pm 0.8$ & $46.3 \pm 0.6$ & 0.423 \\
$D_{98 \%}(G y)$ & $44.7 \pm 0.7$ & $45.1 \pm 0.4$ & 0.435 \\
$H I$ & $0.086 \pm 0.021$ & $0.081 \pm 0.021$ & 0.824 \\
$C l$ & $0.821 \pm 0.034$ & $0.822 \pm 0.031$ & 0.975 \\
\hline
\end{tabular}

Data are expressed as mean \pm SD. OT, ovarian transposition; SD, standard deviation; $D_{2 \%}$, approximate maximum dose; $D_{50 \%}$, median dose; $\mathrm{D}_{98 \%}$, approximate minimum dose; $\mathrm{HI}$, homogeneity index; $\mathrm{Cl}$, conformity index.

Table 3 Comparison of dose parameters for OARs between OT and non-OT patients

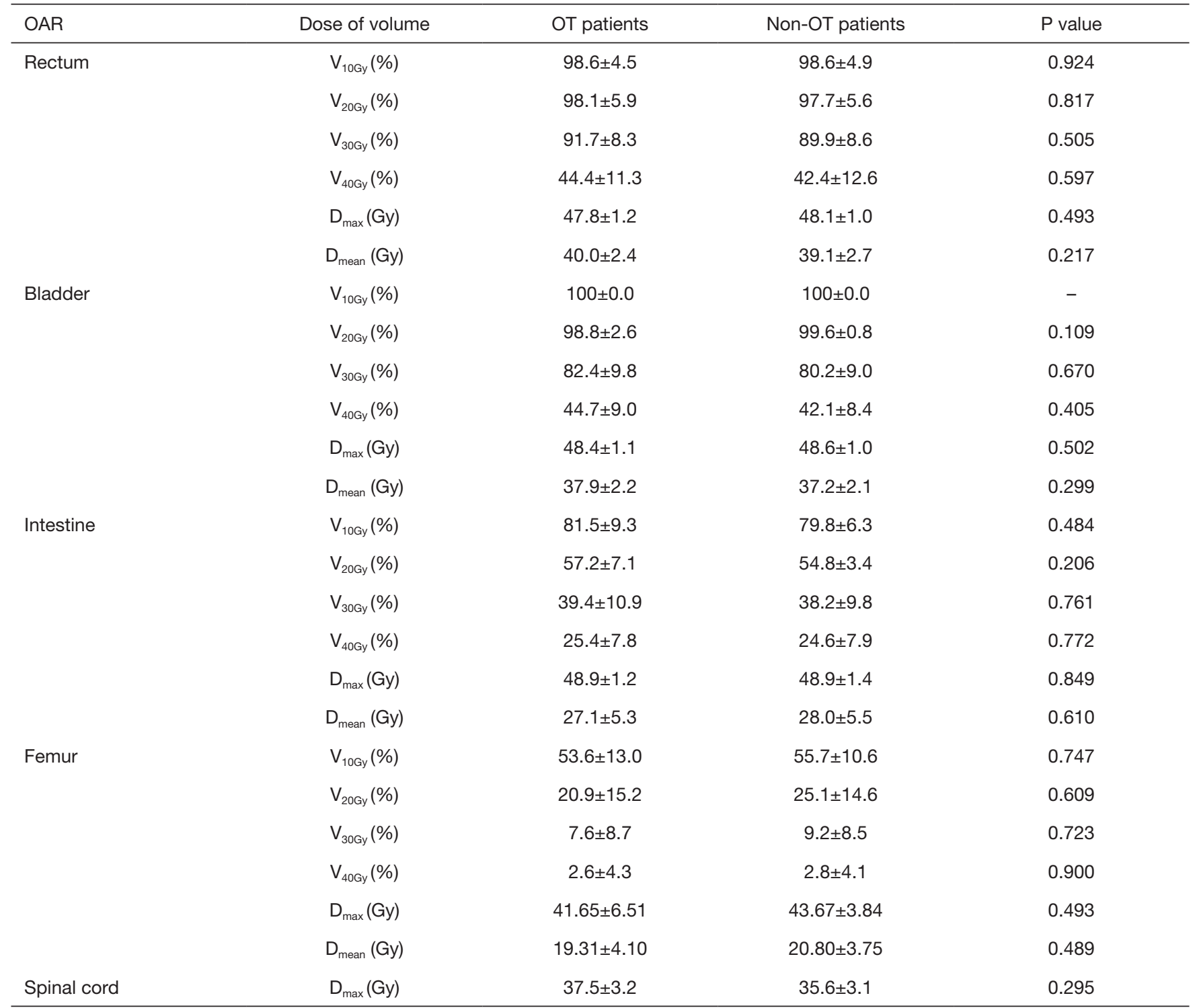

Data are expressed as mean $\pm \mathrm{SD}$. OT, ovarian transposition; SD, standard deviation; OAR, organ at risk; $\mathrm{V}_{\mathrm{xGy}}$ : volume receiving at least $\mathrm{X}$ Gy dose. 
Table 4 Comparison of MUs and treatment times between OT and non-OT patients

\begin{tabular}{lccc}
\hline Item & OT patients & Non-OT patients & P value \\
\hline MUs & $1,040.5 \pm 102.5$ & $894.6 \pm 112.1$ & 0.255 \\
Time (day) & $33.3 \pm 2.4$ & $34.2 \pm 2.7$ & 0.182 \\
\hline
\end{tabular}

Data are expressed as mean \pm SD. OT, ovarian transposition; SD, standard deviation; MUs, monitor units.

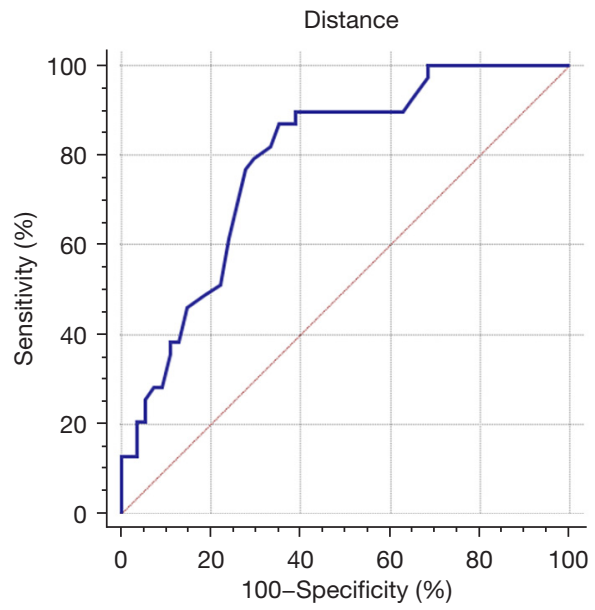

Figure 3 ROC curve determining the optimal cutoff value of the distance for preserved ovarian function. The AUC was $0.787(95 \%$ confidence interval: $0.690-0.865 ; \mathrm{P}<0.001)$, and the optimal cutoff point value was $2.1 \mathrm{~cm}$. ROC, receiver operator characteristic; AUC, area under the curve.

success $(10,20)$.

Although the published rates of preserved ovarian function and complications differ greatly, the safety of the OT procedure remains uncontested, at least for patients with cervical cancer. For example, Pahisa et al. (23) and Han et al. (12) investigated the application of OT in patients with cervical cancer undergoing radiation therapy (4 fields, whole pelvis radiation) and found no cases of ovarian metastasis. Yin et al. and $\mathrm{Lv}$ et al. $(14,15)$ also reported no ovarian metastasis in any OT cases during the follow-up period after postoperative IMRT radiation. Soda et al. noted that the actual rate of these OT ovarian recurrences is rare after patients undergo postoperative radiation (24).

Regarding the impact of OT on cervical cancer prognosis, studies to date have been limited. Turan et al. noted that OT applied to patients with stage Ib cervical cancer did not significantly affect recurrence once postoperative radiotherapy was performed (25) although they did not explain the specific radiation procedure used.

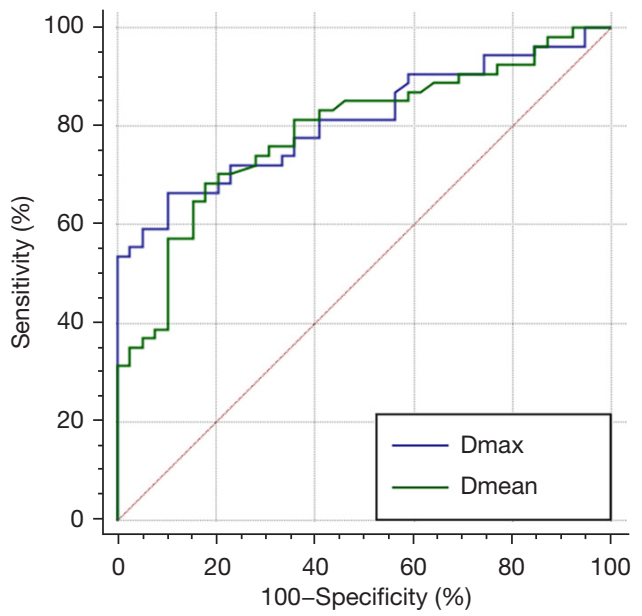

Figure 4 ROC curve determining the optimal cutoff value of ovarian dose for preserved ovarian function. The AUC was 0.812 (95\% confidence interval: $0.717-0.885 ; \mathrm{P}<0.001$ ), and the optimal cutoff point value was $9.8 \mathrm{~Gy}$ for $\mathrm{D}_{\max }$. The AUC was $0.790(95 \%$ confidence interval: $0.693-0.868 ; \mathrm{P}<0.001)$, and the optimal cutoff point value was 4.6 Gy for $\mathrm{D}_{\text {mean }}$. ROC, receiver operator characteristic; AUC, area under the curve; $\mathrm{D}_{\max }$, maximum dose; $\mathrm{D}_{\text {mean }}$, mean dose.

Metastasis of transposed ovaries could be considered negligible in cervical cancer, so the chance of OT altering prognosis is also very slight. This has been verified again in our research, as no cases of ovarian metastases or recurrences have been observed.

Irradiation after OT is undoubtedly correlated with loss of ovarian function; however, the impact differs with the varying radiation therapies. With the emergence of modern radiation therapies, including CT or MRI-based high dose rate (HDR) brachytherapy, as well as current VMAT strategies, the question arises as to whether the ovarian function of premenopausal patients can be maintained using modern sparing techniques in radiation oncology. The issue was first evaluated by Du et al., whose study found that IMRT was the main reason for the absence of ovarian function, which is consistent with previous research (18), but that 
function preservation obtained from constraining the dose of IMRT for OT patients was limited (14). To further reduce the ovarian dose for OT patients, Yoshihiro et al. recently proposed the potentiality of VMAT strategies (16). Based on these facts and our previous experience with VMAT, we designed this study to investigate the preservation of transposed ovarian function in VMAT procedures using OT.

Since patient age has been proven to be repeatedly correlated with the failure of ovarian function, lateral OT is generally recommended for patients younger than 40 years of age $(12,13)$. Although the age range sometimes varies, 40 years is usually advocated as a cutoff value in most published research. Recently, a retrospective control study (20) concluded that OT should be only offered to patients younger than 40 years once pelvic radiation is given. For an accurate assessment of preserved ovarian function as designed for this study, patients older than 40 years were excluded.

The disadvantages of brachytherapy for the survival of transposed ovaries are not conclusive and have resulted in an ongoing debate. For example, one study found that the preservation of ovarian function was only $90 \%$ after OT followed by intravaginal radiation therapy and $60 \%$ after OT followed by intra-vaginal radiation therapy plus external radiation therapy (26). In another study, the same survival of the transposed ovary with or without additional brachytherapy was achieved for OT patients (20). Therefore, patients who had accepted additional brachytherapy were also excluded from the current study.

In our study, 51 young patients were identified. Among these patients, 32 underwent OT, and the dose to the transposed ovaries was limited to as low as possible in the VMAT plan. As reported previously, the ovarian $\mathrm{D}_{\text {mean }}$ was about 1.7 or 4.3 Gy once the transposed ovary was above or below the upper boundary of the PTV, respectively (27). The doses corresponded to the target volumes of $\mathrm{D}_{2 \%}$, $\mathrm{D}_{50 \%}$, and $\mathrm{D}_{98 \%}$. The CI and HI were analyzed initially, showing no significant difference between OT and nonOT patients $(\mathrm{P} \geq 0.05$, Table 2). The analysis revealed that the dose distribution and the target region conformity were not affected by the ovarian dose constraint plan. Interestingly, the HI of the OT patients was slightly higher than that of the non-OT patients (although not significantly so). This difference could be attributed to the fact that the radiation oncologist paid more attention to the plan contouring in OT patients, which in turn increased the plan homogeneity. Furthermore, the irradiation doses of OARs, $\mathrm{V}_{10}, \mathrm{~V}_{20}, \mathrm{~V}_{30}$, and $\mathrm{V}_{40}$, as well as the MUs and the treatment times of OT or non-OT patients, were evaluated, and the results indicated no statistical difference $(\mathrm{P} \geq 0.05$; Table 3). This demonstrated that neither protection of OARs nor treatment efficiency had been sacrificed for the ovarian dose constraint plan.

Over the past 30 years, ovarian insufficiency after OT has varied from $33 \%$ to $100 \%$ in different comparisons (20). Consistent with the previous study results (20), our current study found that $68.8 \%(22 / 32)$ of OT patients retained ovarian function almost 1 year after radiation therapy, which is slightly higher than the results $(41 / 105,39.0 \%)$ of Yin et al. (14), but slightly lower than the results $(56 / 77,72.7 \%)$ of Lv et al. (15). No ovarian metastasis or recurrence was observed in any of the 51 cases. Therefore, OT continues to be a practicable method of maintaining the survival of transposed ovaries during VMAT for postoperative cervical cancer patients.

Previously, the position of the transposed ovary could be guided according to the $2 \mathrm{D}$ box-technique radiotherapy protocol to avoid ovarian failure $(12,13)$. In 2012 and 2014 , it was reported that $77 \%(10 / 13)$ and $71 \%(5 / 7)$ of OT patients, respectively, retained ovarian function after undergoing extending field IMRT with a dose constraint (V7 <50\%) (28,29). A following study in 2017 proposed a dose limitation of IMRT (V7.5 <26\%) in preventing ovarian dysfunction for transposed ovaries (18). Recently, Yin et al. (14) provided newer recommendations $\left(\mathrm{D}_{\max }\right.$ $\leq 9.985 \mathrm{~Gy}, \mathrm{D}_{\text {mean }} \leq 5.32 \mathrm{~Gy}$, and V5.5 $<29.65 \%$ ) in preventing ovarian failure for transposed ovaries. Lv et al. (15) further illustrated the relationship between ovarian location and dose constraint during IMRT for the survival of transposed ovaries. Currently, no specific guideline or recommendation for VMAT is available regarding the dose limit of the transposed ovary.

In the current study, all 19 of the non-OT patients lost ovarian function, indicating that in situ ovarian dysfunction could not be avoided by VMAT. In contrast, the prevention of ovarian insufficiency is possible through limiting the radiation dose delivered by VMAT to the transposed ovaries, as shown in our OT patients. Rather than evaluating the optimum transposition of ovaries (15), we were more interested in exploring the satisfactory distance from the center of a transposed ovary to the PTV margin for the possible preservation of ovarian function. After the ROC curve was applied to this analysis, the minimal distance in VMAT was determined as $2.1 \mathrm{~cm}$ for the preservation of ovarian function in OT patients. These data may provide an initial constraint to maintaining ovarian function using 
VMAT. In addition, we attempted to estimate the radiation dose tolerated by the transposed ovary in these patients. Our results indicated that for the transposed ovary, a $D_{\max }$ of $9.8 \mathrm{~Gy}$ and a $\mathrm{D}_{\text {mean }}$ of $4.6 \mathrm{~Gy}$ may be the cutoff value for the prevention of ovarian function loss. This is comparable with the results of Yin et al. (14). In addition to factors such as patient age and additional radiation elimination, the delivery accuracy of VMAT may provide an explanation as to why the incidence of preserved ovarian function in our study was slightly higher than that in Yin et al.'s study.

As mentioned above, some OT patients still displayed the absence of ovarian function after VMAT though the possible impact factors (e.g., patient age, the additional intravaginal radiation therapy) were taken into consideration at the beginning of this study. Positioning and identification of ovaries during OT may play the same critical role in the prevention of transposed ovarian dysfunction. For those OT patients experiencing loss of ovarian function, part of the transposed ovaries may not have been avoided by contouring in the PTV plan. A possible explanation for this is that the ovaries might have dropped from the transposed point back to their original position or even have fallen into the pelvic irradiation targets (20). To facilitate identification, researchers previously proposed attaching markers to the transposed ovaries (20). Although the markers in our study had been mostly set, in a few cases there were difficulties in recognizing the transposed ovary, either due to the lack of markers or the ovarian migration back to the pelvis (data not shown), and these patients later presented with ovarian dysfunction. Based on our experience, we strongly recommended, if necessary, introducing radiologists into the process of ovary identification, thereby allowing the radiotherapist to optimize the corresponding plan. Moreover, diagnostic MRI scans and ovarian vein-tracking techniques (30) can also be used to accurately identify the unmarked transposed ovaries.

There is a greater likelihood of ovarian dysfunction in OT patients when injury to ovarian vessels occur, with the possible consequence of insufficient vascularization. To date, this problem remains unresolved; differences in surgery types, surgical procedures, and the skills of the different researchers may explain to the varying degrees of success in ovarian survival after OT reported in the literature (31). In our previous study (32), the retention of ovarian function after OT was consistent with that of in situ ovaries, and no statistical difference was found. This probability of injury to ovarian vessels could thus be considered rare in the current study.
The absence of ovarian function significantly impacts quality of life, yet the OT procedure is currently underused. As previously reported (33), OT is used in less than $10 \%$ of cervical cancer patients with tumors who are undergoing pelvic irradiation. Our data highlighted again the potential applications and benefits of OT in preserving ovarian function in young patients with cervical cancer undergoing VMAT therapy.

This study has several limitations. First, there was inadequate follow-up and a lack of comparison for recurrence or metastasis between $\mathrm{OT}$ and non-OT patients. In addition, the small number of patients limited the value of the predictors we obtained for ovarian function preservation. Second, the techniques for radiation delivery have changed from the previous 2D opposite-field radiotherapy to the current VMAT; however, the effect of scattered radiation should still not be ignored. With the increased availability of new techniques (e.g., proton therapy), better OAR sparing may be offered for patients with cervical malignancy (34). Third, the delineation of transposed ovaries prolonged (even doubled; data not shown) the time consumed, resulting in higher physical costs to the radiotherapist. With the emergence of artificial intelligence technology, automatic planning and delineation may provide us with new directions for pelvic irradiation (35).

\section{Conclusions}

In summary, OT is a reliable method for preserving ovarian function in young patients with cervical cancer undergoing postoperative radiotherapy with the VMAT technique, and demonstrates no negative effects on dose distribution, target region conformity, protection of OARs, or treatment efficacy. Specifically, when the distance from the center of the transposed ovary to the PTV margin is more than $2.1 \mathrm{~cm}$, and when the radiation dose is limited to a $D_{\max }$ of less than $9.8 \mathrm{~Gy}$ and a $\mathrm{D}_{\text {mean }}$ of less than $4.6 \mathrm{~Gy}$, the patient's transposed ovarian function may be preserved.

\section{Acknowledgments}

We would like to thank Dr. Jianghai Mei of Toronto, Canada, for her professional help in polishing this paper.

Funding: The study was supported by the Jiangsu Provincial Women and Children Health Research Project (No. F201762), the Clinical Research Fund of the Spark Program for Precision Radiation \& the China International Medical Foundation (No. 2019-N-11-12, HDRS2020030101), the 
Jiangsu Province Association of Maternal and Child Health Grant (FYX202025), and the National Natural Science Foundation of China (No. 81872485).

\section{Footnote}

Reporting Checklist: The authors have completed the STARD reporting checklist. Available at https://dx.doi. org/10.21037/atm-21-2909

Data Sharing Statement: Available at https://dx.doi. org/10.21037/atm-21-2909

Conflicts of Interest: All authors have completed the ICMJE uniform disclosure form (available at https://dx.doi. org/10.21037/atm-21-2909). The authors have no conflicts of interest to declare.

Ethical Statement: The authors are accountable for all aspects of the work in ensuring that questions related to the accuracy or integrity of any part of the work are appropriately investigated and resolved. The study was conducted in accordance with the Declaration of Helsinki (as revised in 2013) and approved by the ethics committee of the Affiliated Cancer Hospital of Nanjing Medical University (No. 2021-006). Individual consent for this retrospective analysis was waived.

Open Access Statement: This is an Open Access article distributed in accordance with the Creative Commons Attribution-NonCommercial-NoDerivs 4.0 International License (CC BY-NC-ND 4.0), which permits the noncommercial replication and distribution of the article with the strict proviso that no changes or edits are made and the original work is properly cited (including links to both the formal publication through the relevant DOI and the license). See: https://creativecommons.org/licenses/by-nc-nd/4.0/.

\section{References}

1. Sung H, Ferlay J, Siegel RL, et al. Global Cancer Statistics 2020: GLOBOCAN Estimates of Incidence and Mortality Worldwide for 36 Cancers in 185 Countries. CA Cancer J Clin 2021;71:209-49.

2. Siegel RL, Miller KD, Fuchs HE, et al. Cancer Statistics, 2021. CA Cancer J Clin 2021;71:7-33.

3. Salman MC, Ozgul N, Yuce K. Widespread recurrence 7 years after radical abdominal trachelectomy for early cervical adenocarcinoma. Case Rep Obstet Gynecol 2015;2015:517496.

4. Li X, Zheng R, Li X, et al. Trends of incidence rate and age at diagnosis for cervical cancer in China, from 2000 to 2014. Chin J Cancer Res 2017;29:477-86.

5. Derks M, Groenman FA, van Lonkhuijzen LRCW, et al. Completing or Abandoning Radical Hysterectomy in Early-Stage Lymph Node-Positive Cervical Cancer: Impact on Disease-Free Survival and Treatment-Related Toxicity. Int J Gynecol Cancer 2017;27:1015-20.

6. Winarto H, Febia E, Purwoto G, et al. The need for laparoscopic ovarian transposition in young patients with cervical cancer undergoing radiotherapy. Int J Reprod Med 2013;2013:173568.

7. Everhov ÅH, Nyberg T, Bergmark K, et al. Hormone therapy after uterine cervical cancer treatment: a Swedish population-based study. Menopause 2015;22:633-9.

8. Moss EL, Taneja S, Munir F, et al. Iatrogenic Menopause After Treatment for Cervical Cancer. Clin Oncol (R Coll Radiol) 2016;28:766-75.

9. Theplib A, Hanprasertpong J, Leetanaporn K. Safety and Prognostic Impacts of Ovarian Preservation during Radical Hysterectomy for Early-Stage Adenocarcinoma and Adenosquamous Cervical Cancer. Biomed Res Int 2020;2020:5791381.

10. Ghadjar P, Budach V, Köhler C, et al. Modern radiation therapy and potential fertility preservation strategies in patients with cervical cancer undergoing chemoradiation. Radiat Oncol 2015;10:50.

11. Batten R, Brown DE. Protection of ovaries from radiation. Lancet 1956;270:939-40.

12. Han SS, Kim YH, Lee SH, et al. Underuse of ovarian transposition in reproductive-aged cancer patients treated by primary or adjuvant pelvic irradiation. J Obstet Gynaecol Res 2011;37:825-9.

13. Hwang JH, Yoo HJ, Park SH, et al. Association between the location of transposed ovary and ovarian function in patients with uterine cervical cancer treated with (postoperative or primary) pelvic radiotherapy. Fertil Steril 2012;97:1387-93.e1-2.

14. Yin L, Lu S, Zhu J, et al. Ovarian transposition before radiotherapy in cervical cancer patients: functional outcome and the adequate dose constraint. Radiat Oncol 2019; 14:100.

15. Lv XJ, Cheng XL, Tu YQ, et al. Association between the location of transposed ovary and ovarian dose in patients with cervical cancer treated with postoperative pelvic radiotherapy. Radiat Oncol 2019;14:230. 
16. Yoshihiro U, Shingo O, Masaru I, et al. Strategies for reducing ovarian dose in volumetric modulated arc therapy (VMAT) for postoperative uterine cervical cancer. Br J Radiol 2018;91:20160777.

17. Wu Y, Zhu B, Han J, et al. A comparative dosimetric study of cervical cancer patients with para-aortic lymph node metastasis treated with volumetric modulated arc therapy vs. 9-field intensity-modulated radiation therapy. Ann Transl Med 2019;7:675.

18. Du Z, Qu H. The relationship between ovarian function and ovarian limited dose in radiotherapy postoperation of ovarian transposition in young patients with cervical cancer. Cancer Med 2017;6:508-15.

19. Flores VA, Pal L. Managing Menopause by Combining Evidence With Clinical Judgment. Clin Obstet Gynecol 2018;61:496-507.

20. Hoekman EJ, Broeders EABJ, Louwe LA, et al. Ovarian function after ovarian transposition and additional pelvic radiotherapy: A systematic review. Eur J Surg Oncol 2019;45:1328-40.

21. Mccall ML, Keaty EC, Thompson JD. Conservation of ovarian tissue in the treatment of carcinoma of the cervix with radical surgery. Am J Obstet Gynecol 1958;75:590600; discussion 600-5.

22. Buekers TE, Anderson B, Sorosky JI, et al. Ovarian function after surgical treatment for cervical cancer. Gynecol Oncol 2001;80:85-8.

23. Pahisa J, Martínez-Román S, Martínez-Zamora MA, et al. Laparoscopic ovarian transposition in patients with early cervical cancer. Int J Gynecol Cancer 2008;18:584-9.

24. Soda I, Ishiyama H, Ono S, et al. Assessment of transposed ovarian movement: how much of a safety margin should be added during pelvic radiotherapy? J Radiat Res 2015;56:354-9.

25. Turan AT, Keskin HL, Dundar B, et al. Ovarian transposition for stage Ib squamous cell cervical cancer lack of effects on survival rates? Asian Pac J Cancer Prev 2013;14:133-7.

26. Morice P, Juncker L, Rey A, et al. Ovarian transposition for

Cite this article as: $\mathrm{Xu} \mathrm{H}$, Guo C, Zhang X, Wu Y, Zhu B, Lu E, Sun Z, He D, Deng F, Lv J, Gong Z. Significance of ovarian transposition in the preservation of ovarian function for young cervical cancer patients undergoing postoperative volumetric modulated radiotherapy. Ann Transl Med 2021;9(23):1717. doi: 10.21037/atm-21-2909 patients with cervical carcinoma treated by radiosurgical combination. Fertil Steril 2000;74:743-8.

27. Guo $\mathrm{C}, \mathrm{Wu} \mathrm{Y}, \mathrm{Zhai} \mathrm{Z}$, et al. Comparison of the ovarian sparing between VMAT and IMRT after ovarian transposition surgery for cervical cancer patients. Chin J Radiol Med Prot 2021;41:414-20.

28. Zhang G, Fu C, Zhang Y, et al. Extended-field intensitymodulated radiotherapy and concurrent cisplatin-based chemotherapy for postoperative cervical cancer with common iliac or para-aortic lymph node metastases: a retrospective review in a single institution. Int J Gynecol Cancer 2012;22:1220-5.

29. Zhang G, He F, Fu C, et al. Definitive extended field intensity-modulated radiotherapy and concurrent cisplatin chemosensitization in the treatment of IB2-IIIB cervical cancer. J Gynecol Oncol 2014;25:14-21.

30. Zhao W, Wang X, Liang Y. Application of 64-slice MSCT with ovarian vein tracking technique in identification of suture-fixed transposed ovaries. Clin Imaging 2018;52:113-6.

31. Al-Badawi IA, Al-Aker M, AlSubhi J, et al. Laparoscopic ovarian transposition before pelvic irradiation: a Saudi tertiary center experience. Int J Gynecol Cancer 2010;20:1082-6.

32. Lv J, Liu ZP, Ren ML. Evaluation of transposed ovarian function in young women with cervical cancer. Journal of Southeast University(Medical Science Edition) 2010;29: 434-49.

33. Selter J, Grossman Becht LC, Huang Y, et al. Utilization of ovarian transposition for fertility preservation among young women with pelvic malignancies who undergo radiotherapy. Am J Obstet Gynecol 2018;219:415-7.

34. Gort EM, Beukema JC, Matysiak W, et al. Interfraction motion robustness and organ sparing potential of proton therapy for cervical cancer. Radiother Oncol 2021;154:194-200.

35. Rhee DJ, Jhingran A, Kisling K, et al. Automated Radiation Treatment Planning for Cervical Cancer. Semin Radiat Oncol 2020;30:340-7. 